CLINICAL STUDY

\title{
AMP-activated protein kinase signaling is upregulated in papillary thyroid cancer
}

Ana Paula Vidal*, Bruno M Andrade ${ }^{1, *}$, Fernanda Vaisman ${ }^{2,3}$, Juliana Cazarin ${ }^{1}$, Luis Felipe Ribeiro Pinto ${ }^{3}$, Marisa Maria Dreyer Breitenbach ${ }^{3}$, Rossana Corbo ${ }^{2,3}$, Adriana Caroli-Bottino, Fernando Soares ${ }^{4}$, Mario Vaisman ${ }^{2}$ and Denise P Carvalho ${ }^{1}$

Serviço de Anatomia Patológica do Hospital Universitário Clementino Fraga Filho, Universidade Federal do Rio de Janeiro, Avenida Rodolpho Paulo Rocco 255, Ilha do Fundão, Rio de Janeiro, Brazil, ${ }^{1}$ Laboratório de Fisiologia Endócrina, Instituto de Biofísica Carlos Chagas Filho, Universidade Federal do Rio de Janeiro, Avenida Carlos Chagas Filho, 373 - CCS - Bloco G - G1060, Ilha do Fundão, Rio de Janeiro, Brazil, ${ }^{2}$ Serviço de Endocrinologia do Hospital

Universitário Clementino Fraga Filho, Universidade Federal do Rio de Janeiro, Avenida Rodolpho Paulo Rocco 255, Ilha do Fundão, Rio de Janeiro, Brazil, ${ }^{3}$ Instituto Nacional de Câncer, Praça da Cruz Vermelha 23, Centro, Rio de Janeiro, Brazil and ${ }^{4}$ Serviço de Patológica, Hospital A.C. Camargo, Rua Professor Antonio Prudente 211, Liberdade, São Paulo, Brazil

(Correspondence should be addressed to D P Carvalho; Email: dencarv@bifo.ufrj.br)

*(A P Vidal and B M Andrade contributed equally to this work)

\begin{abstract}
AMP-activated protein kinase (AMPK) is activated by the depletion in cellular energy levels and allows adaptive changes in cell metabolism and cell survival. Recently, our group described that AMPK plays an important role in the regulation of iodide and glucose uptake in thyroid cells. However, AMPK signaling pathway in human thyroid carcinomas has not been investigated so far.

Objective: To evaluate the expression and activity of AMPK in papillary thyroid carcinomas.

Methods: We examined total and phosphorylated AMPK (tAMPK and pAMPK) and phosphorylated acetyl-CoA-carboxylase (pACC) expressions through imunohistochemistry, using a tissue microarray block composed of 73 papillary thyroid carcinomas (PAP CA) or microcarcinomas (PAP MCA) and six adenoma (AD) samples from patients followed at the Federal University Hospital. The expression levels were compared with the non-neoplastic tissues from the same patient. Two different pathologists analyzed the samples and attributed scores of staining intensity and the proportion of stained cells. A total index was obtained by multiplying the values of intensity and the proportion of stained cells (INTxPROP).

Results: tAMPK, pAMPK, and pACC showed a predominant cytoplasmic staining in papillary carcinomas, adenomas, and non-neoplastic thyroid tissues. However, the intensity and the proportion of stained cells were higher in carcinomas, so that a significant increase was found in the INTxPROP score both in PAP CA and PAP MCA, when compared with their respective controls.

Conclusion: Our results show unequivocally that AMPK pathway is highly activated in papillary thyroid carcinomas; however, more studies are necessary to understand the pathophysiological significance of AMPK activation in thyroid carcinogenesis.
\end{abstract}

European Journal of Endocrinology 169 521-528

\section{Introduction}

AMP-activated protein kinase (AMPK) is a metabolic stress-sensing cytoplasmic enzyme composed of an $\alpha$-catalytic and two regulatory subunits $(\beta$ and $\gamma)(1)$. Stresses that deplete energy and increase the intracellular AMP-to-ATP ratio induce allosteric activation of AMPK $(1,2)$, promoting conformational changes that make the enzyme a better substrate for upstream kinases, which phosphorylate its Thr-172 residue and further activate AMPK. In its activated state, AMPK shuts down processes that consume energy and upregulates energy-producing pathways in an attempt to restore intracellular ATP levels $(1,2,3)$. One of the well-described effects of AMPK is the inhibition of acetylCoA-carboxylase (ACC), an enzyme responsible for the conversion of acetyl-CoA to malonyl-CoA in de novo fatty acid biosynthesis. Malonyl-CoA is a potent inhibitor of carnitine palmitoyl transferase-1, responsible for the transport of long-chain fatty acid to mitochondrial matrix. The reduction of malonyl-CoA induced by AMPK activation thus favors fatty acid translocation into the mitochondria to be oxidized, in an attempt to restore intracellular ATP levels $(1,2,3,4)$. Activation of AMPK can be evaluated by the use of a specific antibody that recognizes the catalytic subunits of AMPK when phosphorylated in Thr-172 residue (4). Also, a direct inference of AMPK activity is obtained through the 
analyses of the phosphorylation in Ser-79 of its downstream target, ACC (4). In its activated state, AMPK allows cellular adaptive changes in order to maintain growth, differentiation, and metabolism under conditions of low intracellular energy availability $(1,2,3,4,5)$.

Recently, we described the involvement of AMPK signaling in the regulation of iodide and glucose uptake in rat thyrocytes, both in vitro and in vivo $(3,6)$. We showed that the AMPK activator, 5-aminoimidazole-4-carboxamideribonucleoside (AICAR), decreases iodide uptake (in vitro and in vivo) and NIS protein and mRNA content in thyroid cells (4). Also, using the same methodological approach, we have recently demonstrated that AICAR produces a concentration-dependent increase in glucose uptake by thyroid PCCL3 cells (6). It is important to notice/emphasize that tumor cells are submitted to metabolically stressful conditions and a shift toward glucose metabolism, known as the 'Warburg Effect' occurs. Therefore, it is tempting to speculate whether AMPK activation could be implicated in this phenomenon.

Although the role of AMPK signaling in cancer cells is not completely understood, some evidence suggests that AMPK activation leads to anti-proliferative effects, with G1-S phase cell cycle arrest $(7,8,9,10)$. Further evidence for AMPK anti-proliferative effects on tumor cells relies on the fact that the major kinase that phosphorylates and activates AMPK has been identified to be the tumor suppressor kinase LKB1 (9). The previous findings showing that the oral anti-diabetic drug, metformin, inhibits proliferation of epithelial cells derived from breast, prostate, and ovarian cancers, effects that require both LKB1 and AMPK, are consistent with the concept that AMPK pathway might be implicated in tumor cell biology $(1,11,12,13)$. Also, several epidemiological studies demonstrated that the chronic use of metformin is associated with a lower incidence of cancer $(1,13)$. On the one hand, activated AMPK shows anti-proliferative effects on tumor cells; on the other hand, it leads to tumor cell survival, as cell adaption to adverse conditions, such as glucose deprivation and hypoxia, might require AMPK activation $(1,14,15)$.

Differentiated thyroid carcinomas (DTC) are slowgrowing and usually curable forms of thyroid cancer (16). The adequate intervention for this type of cancer includes the combined effects of surgery, radioiodine ablation, and thyroid-stimulating hormone suppressive therapy $(17,18,19)$. However, tumor recurrence can occur in about $20-30 \%$ of patients with DTC, which reinforces the importance of unraveling novel targets for thyroid cancer diagnosis and treatment $(20,21,22)$. Previous reports show that thyroid tumor progression is accompanied by increased glucose uptake detected by 18F-fluorodeoxiglucose positron emission tomography (FDG-PET) and decreased radioiodide uptake ability (23). Thus, based on our previous findings in normal thyroid cells, we hypothesized that the expression and activation of AMPK could be modulated in thyroid tumors.
Hence, our objective in this study was to evaluate the expression of total AMPK (tAMPK), phosphorylated AMPK (pAMPK), and phosphorylated ACC in papillary thyroid carcinomas and microcarcinomas, in relation to adenomas and the non-neoplastic tissue (NNT) of the same patient. We herein describe for the first time that the AMPK pathway is significantly upregulated in papillary thyroid carcinomas, while normally expressed in benign hyperplastic lesions of the thyroid.

\section{Subjects and methods}

\section{Patients}

This retrospective study used paraffin-embedded tissue blocks from 79 patients, accompanied at the Clementino Fraga Filho University Hospital from the Federal University of Rio de Janeiro, who underwent resection of papillary thyroid carcinomas $(n=73)$ or thyroid follicular adenomas $(n=6)$ between 1998 and 2008 . After obtaining approval from the institutional review board, we retrospectively reviewed the electronic medical records of the patients. Each slide case was reviewed by the same two pathologists and classified according to the classification of the World Health Organization (22). The patients consisted of 12 men and 61 women, and the mean patient age at the time of surgery was $45.2 \pm 15.3$ years old (range: $14-87$ years old). All available clinical, pathological, and follow-up data were collected from our database, reviewed and updated for all patients.

\section{Tissue microarray}

Thyroid tissue specimens were fixed in 10\% buffered formaldehyde solution and embedded in paraffin. We reviewed the available slides and selected the paraffinembedded tissue blocks. The diagnosis of carcinomas was confirmed using hematoxylin- and eosin (HE)stained sections, following standard criteria according to the classification of the World Health Organization (22). Using a manual tissue microarray (TMA) instrument (Beecher Instruments, Sun Prairie, WI, USA), two blocks of high-density TMA were designed to include at least two samples of 57 papillary thyroid carcinomas $(>1 \mathrm{~cm}$, CAPAP) distributed as 50 classical and seven follicular subtypes, 16 papillary microcarcinomas ( $<1 \mathrm{~cm}$, MCAPAP), and six follicular adenomas. All the samples were analyzed in relation to their corresponding NNT. A total of 236 spots were analyzed.

\section{Immunohistochemistry}

AMPK $\alpha$, phospho-AMPK $\alpha$ (Thr-172)-(40H9), and phospho-acetyl-CoA carboxylase (Ser-79) primary antibodies were purchased from Cell Signaling Technology (Berverly, MA, USA). The antibodies were 
previously tested for immunohistochemistry analysis in paraffin-embedded tissue, as mentioned by the manufacturer. Optimal staining conditions such as epitope unmasking, antibody titer, and incubation and visualization methods were validated in our service using conventional whole tissue sections and TMA fragments. For the immunostaining reaction, DAKO autostainer using Vectastatin ABC kits (Vector Labs, Peterborough, UK) were used according to the manufacturer's protocol. The antigen retrieval was carried out in citrate buffer ( $\mathrm{pH}$ 6.0) vaporized at $95-98^{\circ} \mathrm{C}$ for $20 \mathrm{~min}$. Tissues were subject to blocking of endogenous peroxidase activity with 3\% hydrogen peroxide. Sections were then incubated with the primary antibody overnight, at $4{ }^{\circ} \mathrm{C}$, in the dilution of $1: 50$. Ductal breast carcinoma was used as positive and negative controls of the reactions. Omitting the primary antibody was carried out to provide negative controls. Following the incubation with the specific primary antibodies, sections were incubated with either biotinylated antirabbit, anti-sheep, or anti-mouse antibodies for $30 \mathrm{~min}$, followed by Vectastain Elite $\mathrm{ABC}$ reagent for another $30 \mathrm{~min}$. Liquid diaminobenzidine (Dako, Glostrup, Denmark) was used as a chromogenic agent for $5 \mathrm{~min}$ and sections were counterstained with Mayer's hematoxylin. After each immunostaining step, the slides were briefly washed in PBS buffer, pH 7.6.

Two independent pathologists (V A P and C B A) analyzed the immunostaining reactions. The observers were unaware of the clinical history and the follow-up of the patients. Each staining was assessed using a scoring system based on the Quick Score Method (23). Immunoreactivity was scored semiquantitatively for both the staining intensity and the proportion of cytoplasmic cell staining. Intensity evaluation used scores that ranged from 0 to $3(0=$ negative; $1=$ light; $2=$ moderate; $3=$ strong $)$ and the proportion scores ranged from 1 to $6(1=0-4 \% ; 2=5-20 \% ; 3=21-40 \%$; $4=41-60 \% ; 5=61-80 \% ; 6=81-100 \%)$. The two scores were then multiplied to obtain a total index of staining. Average score was taken as the final score between duplicate spots of the same patient. The staining analyses were scored at the magnification of $40 \times$ to estimate the proportion of positive cells. The two pathologists, using a double-headed microscope, reevaluated all cases with discrepant scores and a consensus was reached.

\section{Statistical analysis}

The statistical analysis was done using GraphPad Prism (La Jolla, CA, USA) 5.0 software. For the histological quantification of intensity and proportion, we analyzed data using the nonparametric Wilcoxon matched pairs test for the distribution frequency of the scores. Data shown in Table 1 correspond to the differences in ranks in two conditions (NNT and CAPAP; NNT and MCAPAP). Data are expressed in number and percentiles of those
Table 1 Difference between paired samples of NNT and CAPAP/MCAPAP in intensity score calculated by Wilcoxon test for total AMPK, phospho-AMPK, and phospho-ACC.

\begin{tabular}{|c|c|c|c|c|}
\hline & $\begin{array}{c}\text { Difference } \\
\text { between } \\
\text { NTT and } \\
\text { CAPAP } n(\%)\end{array}$ & $P$ & $\begin{array}{c}\text { Difference } \\
\text { between } \\
\text { NTT and } \\
\text { MCAPAP } n(\%)\end{array}$ & $\boldsymbol{P}$ \\
\hline \multicolumn{5}{|l|}{ Total AMPK } \\
\hline Reduced & $17(30)$ & \multirow{3}{*}{0.04} & $6(37)$ & \multirow{3}{*}{ NS } \\
\hline Maintained & $33(58)$ & & $8(50)$ & \\
\hline Increased & $7(12)$ & & $2(13)$ & \\
\hline \multicolumn{5}{|c|}{ Phospho-AMPK } \\
\hline Reduced & $8(14)$ & \multirow{3}{*}{0.01} & $3(18)$ & \multirow{3}{*}{ NS } \\
\hline Maintained & $26(46)$ & & $7(44)$ & \\
\hline Increased & $23(40)$ & & $6(38)$ & \\
\hline \multicolumn{5}{|l|}{ Phospho-ACC } \\
\hline Reduced & 7 (12) & \multirow{3}{*}{0.001} & $1(6)$ & \multirow{3}{*}{0.007} \\
\hline Maintained & $27(48)$ & & $5(31)$ & \\
\hline Increased & $23(40)$ & & $10(63)$ & \\
\hline
\end{tabular}

NS, non-significant.

conditions in the ranks of the test that were denominated as reduced, maintained, and increased. For the analysis of the total index obtained in tumor and NNT, data were expressed as mean \pm s.e.m. and analyzed using the Wilcoxon matched pairs test. $P$ values $<0.05$ were considered statistically significant.

\section{Results}

\section{Immunohistochemistry analysis of tAMPK, PAMPK, and pACC in CAPAP of thyroid}

tAMPK, pAMPK, and phospho-ACC (pACC) showed a cytoplasmic staining in papillary carcinomas and nonneoplastic thyroid tissues (Fig. 1, center and right columns). Apart from cytoplasmic staining, membrane and nuclear stainings were also present. Ductal breast carcinoma was used as positive and negative controls of the reactions (Fig. 1, left column). Strong and diffuse staining was observed for both tAMPK and pAMPK (Fig. 1, first and second lines) in the majority of papillary carcinomas. Most spots of non-neoplastic thyroid tissue showed a focal positivity for either tAMPK or pAMPK (Fig. 1, first and second lines). Diffuse staining was observed for pACC in papillary carcinoma, while a focal pattern was observed in NNT (Fig. 1, third line). Increased AMPK phosphorylation signal was seen in the vast majority of papillary thyroid cancer specimens, as a diffuse and strong staining in the cytoplasm compared with the focal expression in normal epithelium with a significant positive association.

\section{Comparison of tAMPK, pAMPK, and pACC expression between CAPAP and MCAPAP of thyroid}

In order to quantify the expression pattern of AMPK pathway in thyroid cancer, we analyzed the intensity 


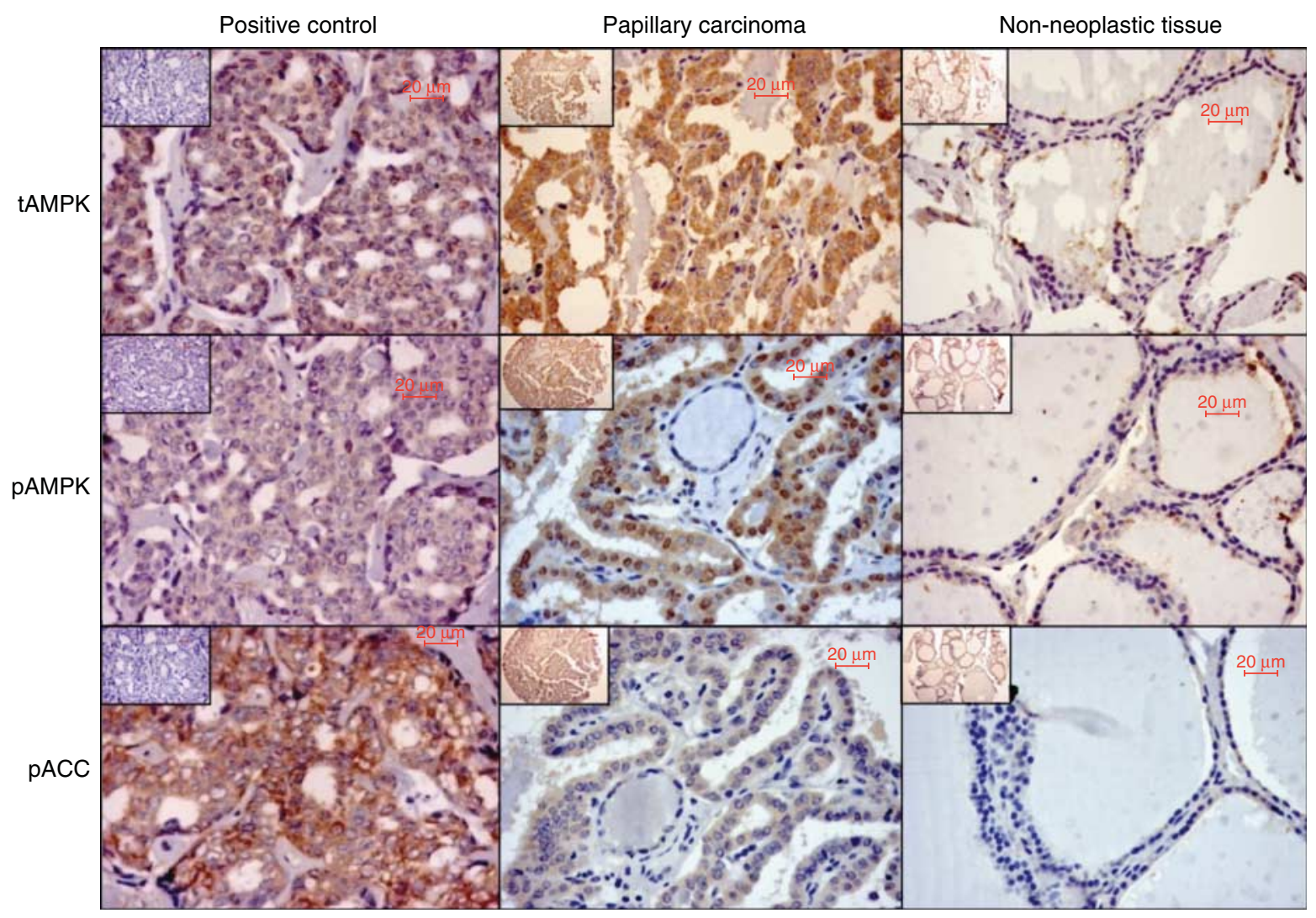

Figure 1 Photomicrographs of papillary thyroid carcinomas and non-tumoral tissue. Photomicrographs in the left column are representatives of positive immunostaining controls $(40 \times)$ with breast cancer samples with a corresponding negative control spot $(40 \times)$ in total AMPK, phospho-AMPK, and phospho-ACC; center column is representative of papillary carcinoma immunostaining in $40 \times$ magnification and the respective spots at 10X magnification; right column represents non-neoplastic tissue (NNT). Photomicrographs on the first line are representative of total AMPK immunostaining in controls, papillary carcinoma, and NNT; photomicrographs in the centerline are representative of phospho-AMPK and in the third line they represent phospho-ACC.

and proportion of immunostaining, which represent the level and number of cells expressing tAMPK, pAMPK, and pACC.

Table 1 shows the frequency distribution of staining intensity for tAMPK, pAMPK, and pACC. We observed a significantly different pattern of distribution of intensity score for tAMPK, pAMPK, and pACC in CAPAP vs their respective NNT. The pattern of distribution indicates that the majority of CAPAP samples maintained a similar intensity score when paired with their NNT, and the second most frequent pattern of intensity score was an increased staining in CAPAP when compared with NNT. Interestingly, there was no significant difference when tAMPK and pAMPK intensity scores were compared with NTT and MCAPAP, although the pACC intensity score was more frequently found to increased rank in MCAPAP when compared with their NTT counterparts.

Figure $2 \mathrm{~A}, \mathrm{~B}$, and $\mathrm{C}$ shows the results of proportion scores of tAMPK, pAMPK, and pACC in CAPAP, MCAPAP, and NNT. There are significantly higher frequency distributions for all the three targets in cancers when compared with the respective normal tissue, clearly showing an increase in score 6 in cancer samples, for all the proteins analyzed. Corroborating with the description of heterogeneity in normal thyroid follicles, all the different scores of proportion for AMPK expression were detected in NNT. These results suggest that AMPK also plays a physiological role in human thyroids, although this has to be confirmed. In papillary thyroid cancers however, tAMPK, pAMPK, and pACC the proportion of stained cells with a score of 6 predominated, representing more than $95 \%$ of cancer cells. This finding suggests that AMPK may probably be implicated in thyroid cancer cell transformation.

\section{Comparing intensity and proportion of tAMPK, PAMPK and PACC in cancer vs NNT of the same patient}

To further confirm the increased expression and activity of AMPK in thyroid cancer, we multiplied the scores of intensity and proportion and compared the values of the total index found in paired samples of cancers and NNT of the same patient. As shown in Fig. 3A, B, and C, the expression of tAMPK, pAMPK, and pACC is significantly 

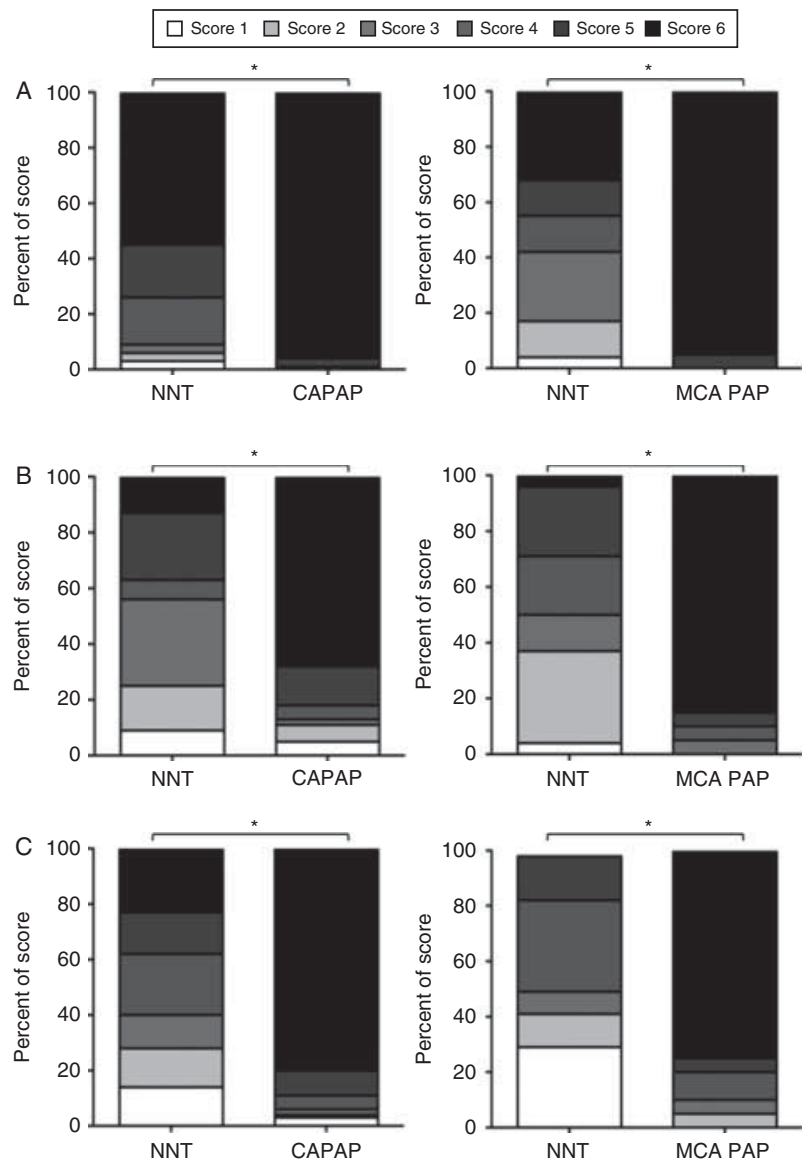

Figure 2 Distribution pattern of the proportion of total AMPK, phospho-AMPK, and phospho-ACC in papillary $(n=57)$ and micropapillary $(n=16)$ carcinomas and their respective non-neoplastic thyroid tissue samples. The staining proportion was scored using a range from 1 to 6 regarding the number of cells stained in each TMA spot. (A) Total AMPK; (B) phospho-AMPK; (C) phospho-ACC. Results are shown as percent of the total number of cases in each score. ${ }^{*} P<0.0001$ vs control ( $\chi^{2}$ test).

increased in both CAPAP and MCAPAP compared with their respective NNT samples. These results initially suggest that AMPK is not only more expressed but also highly active in thyroid cancer cells than in NNT. In order to correlate the increase in expression and activity of AMPK with carcinogenesis, we analyzed six samples of thyroid adenomas. Interestingly, there was no significant difference in the expression of tAMPK, pAMPK, and pACC in adenomas when compared with the NNT of the same patients (Fig. 3C).

\section{Indirect analysis of AMPK activity through the ratio between pACC and the relationship between PAMPK and tAMPK expression in cancers}

In order to evaluate the activity of AMPK in human papillary thyroid cancer, we calculated the ratio between pACC and AMPK phosphorylation rate (pAMPK/tAMPK). This analysis is necessary in order to exclude the possibility that the observed increase in pAMPK could only be related to the increase in tAMPK expression and not to a greater activity of this kinase. Indeed, Fig. 4A and $\mathrm{B}$ showed and confirmed that papillary and micro-papillary cancer samples have increased AMPK activity compared with their respective NNT and adenomas (Fig. 4C).

\section{Discussion}

In this study, we describe for the first time that the metabolic sensor AMPK is expressed in normal human thyroid gland and that its expression and activity are significantly higher in papillary thyroid carcinomas. As shown herein, a strong and diffuse cytoplasmic staining is observed for tAMPK and pAMPK in the majority of papillary carcinomas in contrast with the focal positivity found in non-tumor thyroid tissue cells. It is interesting to notice that in the previous studies regarding the molecular signature of PAPCA through microarray, AMPK mRNA has not been identified as differentially expressed. The differences between those studies and our present results could be related to the different sensitivity of the methods used and/or due to increased translation of AMPK mRNA, with no differences in its expression levels (24).

A significantly higher expression and activity of AMPK were found in thyroid cancer cells in relation to a higher total index (intensity $\times$ proportion) of immunostaining. Furthermore, there was a significant difference in the proportion of cells stained for the three proteins: tAMPK, pAMPK, and pACC, suggesting that when we compare normal vs papillary thyroid carcinomas, a strong stimulus for AMPK expression and activation is observed.

Although the cytoplasmic staining in both NNT and papillary carcinoma thyroid tissues were predominant, we also observed membrane and nuclear staining (Fig. 1, center and right columns). The relevance of these findings, however, is not known. It has been demonstrated that AMPK $\alpha 2$ is localized in the nuclei of many cells and might be involved in the regulation of gene expression (25). The physiological significance of the increase in AMPK expression and activity still has to be elucidated. Recently, Faustino et al. (2012) (25) published a study describing the pattern of expression and activity of mTOR in thyroid cancer lesions. The authors showed that the PI3K/AKT/mTOR pathway is activated and that there is a correlation between this pathway activation and $B R A F^{V 600 E}$ mutations in thyroid carcinomas (26). The interrelationship between AMPK and mTOR is well described for tissues other than the thyroid (27). Our group has previously described that mTOR plays an important role in the regulation of iodide uptake in rat thyrocytes $(28,29)$. Although not 

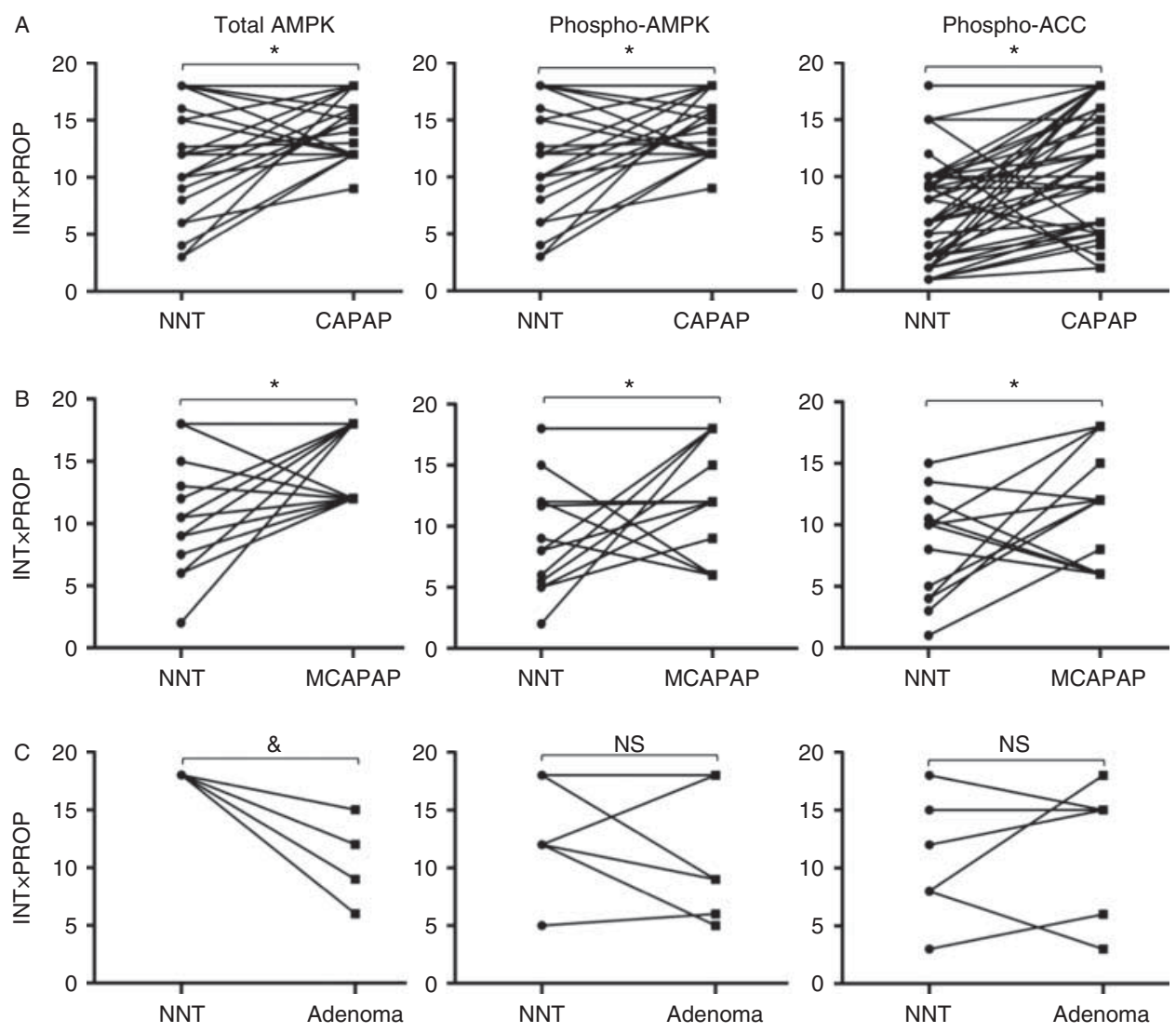

Figure 3 Expression pattern of total AMPK, phospho-AMPK, and phospho-ACC in papillary $(n=57)$ and micro-papillary ( $n=16$ ) carcinomas, adenomas $(n=6)$, and non-neoplastic thyroid paired samples. The antigen expression is represented by a score that corresponds to the multiplication of the value attributed to the staining intensity (from 0 to 3 ) by the value attributed to the staining proportion (0-6) (maximum value is therefore $3 \times 6=18$ ), comparing carcinoma vs the non-neoplastic tissue of the same patient. (A) Papillary carcinoma; (B) micro-papillary carcinoma; (C) adenoma. Results are shown by mean \pm S.E.M. ${ }^{\star} P<0.001$ vs control; ${ }^{\&} P<0.05$ vs control (Wilcoxon paired test).

yet demonstrated for the thyroid tissue, under normal physiological conditions, AMPK activation inhibits mTOR through different mechanisms (27). Nevertheless, as our study clearly demonstrates increased expression and activity of AMPK in papillary thyroid carcinomas, and taking into consideration the previous report of Faustino et al. (2012) (25), one might speculate that at least in papillary thyroid carcinomas both MTOR and AMPK pathways are stimulated. The apparent controversy might be explained by the finding of Esteve-Puig et al. (2009) (27), who showed that $B R A F^{\mathrm{V} 600 \mathrm{E}}$ mutation in melanoma cells leads to uncoupling in LKB1-AMPK pathway, also changing its relationship with the mTOR signaling pathway (30). However, the relationship between AMPK and mTOR pathways in normal thyroid cells and in thyroid cancers needs to be evaluated in future studies, preferably using fresh tumor samples and a more quantitative analysis.

Previous studies from our group have described that AMPK activation in normal thyroid gland and PCCL3 rat thyroid cell lineage leads to decreased iodide uptake and increased glucose uptake $(2,5)$. This phenomenon (so called 'flip-flop') is commonly observed in thyroid oncology and seems to be well correlated with gain in tumor aggressiveness $(20,21,31)$. Therefore, it is tempting to speculate whether changes in AMPK expression could play a role in thyroid tumor progression, although this has to be further evaluated. Buzzai et al. (2005) (14) demonstrated that AMPK is necessary for the survival of $\mathrm{LN}-229$ cells during glucose deprivation protocol, indicating that this pathway might be involved in the control of tumor cell survival under stressful conditions. No matter the significance of AMPK activation for cancer prognosis, we can conclude that the higher expression and activity of AMPK in well-differentiated thyroid cancer could be related to the lower iodide uptake ability of thyroid cancer cells. As some clinical data point to the possibility that metformin - an AMPK agonist - might reduce cancer incidence, it is important to better understand the role of this kinase in tumorigenesis, cancer progression, and the reduced iodide uptake that impairs radioiodine therapeutically approach. 

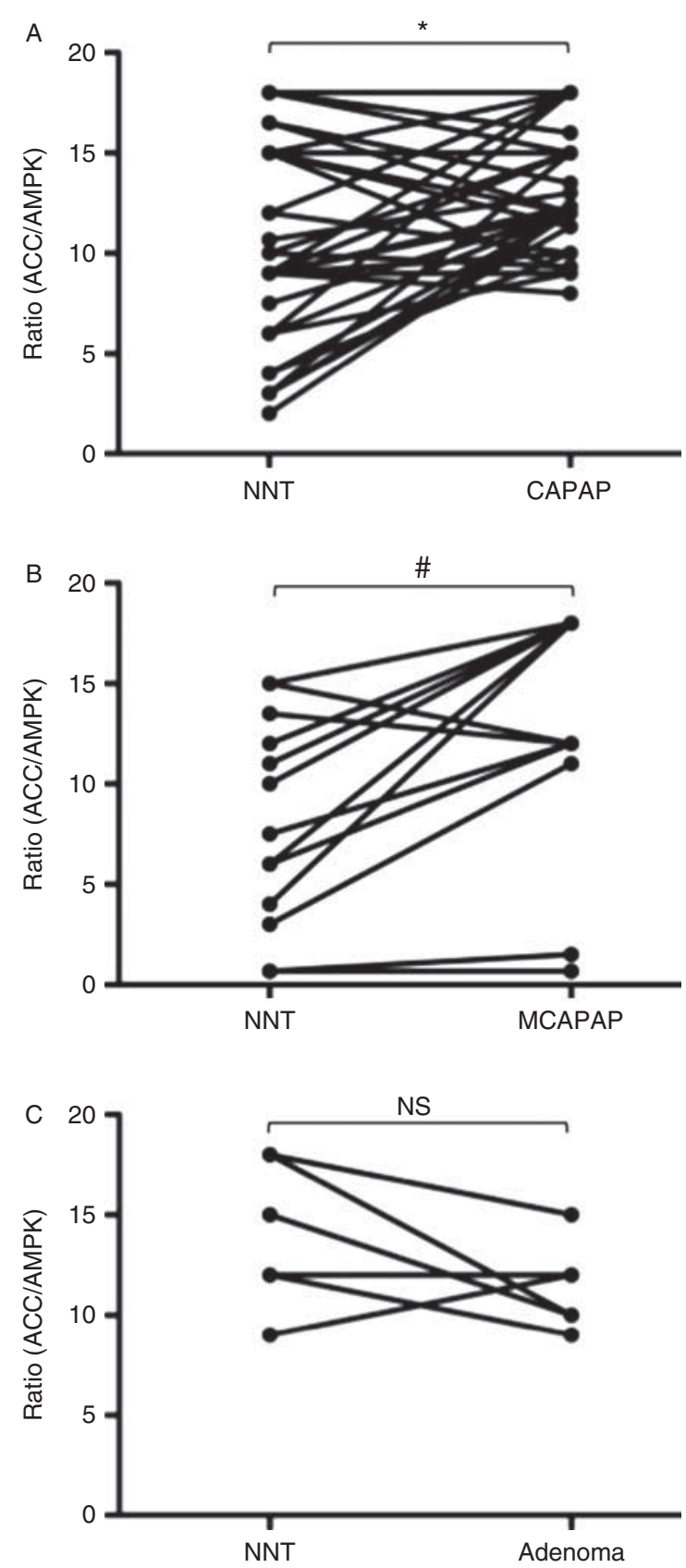

Figure 4 Ratio of expression of phospho-ACC in relation to (total AMPK/phospho-AMPK) in papillary $(n=57)$ and micro-papillary $(n=16)$ carcinomas, adenomas $(n=6)$, and non-neoplastic thyroid paired samples. The ratio of expression was calculated through the division of the value attributed to phospho-ACC and the ratio of phospho-AMPK and total AMPK pattern of expression, comparing carcinoma vs normal tissue of the same patient. (A) Papillary carcinoma; (B) micro-papillary carcinoma; (C) adenoma. Results are shown by mean \pm s. E.M. . ${ }^{\star} P<0.0001$ vs control; ${ }^{\#} P<0.001$ vs control; NS, not significant (Wilcoxon paired test).

In conclusion, our data show that AMPK expression and phosphorylation are increased in papillary thyroid cancer specimens when compared with the non-neoplastic counterpart tissues and benign lesions. This finding suggests that AMPK may probably be implicated in thyroid cancer cell transformation. More data are now required to give us a comprehensive understanding about the role of AMPK pathway in thyroid carcinoma.

\section{Declaration of interest}

The authors declare that there is no conflict of interest that could be perceived as prejudicing the impartiality of the research reported.

\section{Funding}

This work was supported by grants from Fundação Carlos Chagas Filho de Amparo a Pesquisa no Estado do Rio de Janeiro (FAPERJ), Coordenação de Aperfeiçoamento de Pessoal de Nível Superior (CAPES), Conselho Nacional para o Desenvolvimento Científico e Tecnológico (CNPq), Instituto Nacional de Ciência e Tecnologia para Pesquisa Translacional em Saúde e Ambiente (INPeTAm).

\section{Acknowledgements}

The authors thank Heliomar Pereira Marcos for the contribution in immunostaining experiments.

\section{References}

1 Dandapani M \& Hardie DG. AMPK: opposing the metabolic changes in both tumour cells and inflammatory cells? Biochemical Society Transactions 201341 687-693. (doi:10.1042/BST201 20351)

2 Hardie DG. AMP-activated protein kinase: an energy sensor that regulates all aspects of cell function. Genes and Development 2011 25 1895-1908. (doi:10.1101/gad.17420111)

3 Andrade BM, Araujo RL, Perry RL, Souza EC, Cazarin JM, Carvalho DP \& Ceddia RB. A novel role for AMP-kinase in the regulation of the $\mathrm{Na}^{+} / \mathrm{I}^{-}$-symporter and iodide uptake in the rat thyroid gland. American Journal of Physiology. Cell Physiology 2011 300 C1291-C1297. (doi:10.1152/ajpcell.00136.2010)

4 Gaidhu MP \& Ceddia RB. Remodeling glucose and lipid metabolism through AMPK activation: relevance for treating obesity and type 2 diabetes. Clinical Lipidology 20094 465-477. (doi:10.2217/clp. 09.30)

5 Kahn BB, Alquier T, Carling D \& Hardie DG. AMP-activated protein kinase: ancient energy gauge provides clues to modern understanding of metabolism. Cell Metabolism 2005 1 15-25. (doi:10.1016/j.cmet.2004.12.003)

6 Andrade BM, Cazarin J, Zancan P \& Carvalho DP. AMP-activated protein kinase upregulates glucose uptake in thyroid PCCL3 cells independent of thyrotropin. Thyroid 201222 1063-1068. (doi:10.1089/thy.2012.0041)

7 Rattan R, Giri S, Singh AK \& Singh I. 5-Aminoimidazole-4carboxamide-1- $\beta$-D-ribofuranoside inhibits cancer cell proliferation in vitro and in vivo via AMP-activated protein kinase. Journal of Biological Chemistry 2005280 39582-39593. (doi:10.1074/jbc. M507443200)

8 Luo Z, Zang M \& Guo W. AMPK as a metabolic tumor suppressor: control of metabolism and cell growth. Future Oncology 20106 457-470. (doi:10.2217/fon.09.174)

9 Shackelford DB \& Shaw RJ. The LKB1-AMPK pathway: metabolism and growth control in tumor suppression. Nature Reviews. Cancer 20099 563-575. (doi:10.1038/nrc2676)

10 Jones RG, Plas DR, Kubek S, Buzzai M, Mu J, Xu Y, Birnbaum MJ \& Thompson CB. AMP-activated protein kinase induces a p53dependent metabolic checkpoint. Molecular Cell 200518 283-293. (doi:10.1016/j.molcel.2005.03.027) 
11 Grisouard J, Dembinski K, Mayer D, Keller U, Müller B \& ChristCrain M. Targeting AMP-activated protein kinase in adipocytes to modulate obesity-related adipokine production associated with insulin resistance and breast cancer cell proliferation. Diabetology $\mathcal{E}$ Metabolic Syndrome 201120 3-16. (doi:10.1186/1758-59963-16)

12 Hadad SM, Baker L, Quinlan PR, Robertson KE, Bray SE, Thomson G, Kellock D, Jordan LB, Purdie CA, Hardie DG et al. Histological evaluation of AMPK signalling in primary breast cancer. BMC Cancer 20099 307. (doi:10.1186/1471-2407-9-307)

13 Gallagher EJ \& LeRoith D. Diabetes, cancer, and metformin: connections of metabolism and cell proliferation. Annals of the New York Academy of Sciences 20111243 54-68. (doi:10.1111/ j.1749-6632.2011.06285.x)

14 Buzzai M, Bauer DE, Jones RG, Deberardinis RJ, Hatzivassiliou G, Elstrom RL \& Thompson CB. The glucose dependence of Akt-transformed cells can be reversed by pharmacologic activation of fatty acid $\beta$-oxidation. Oncogene $2005 \mathbf{2 4} 4165-4173$. (doi:10.1038/sj.onc.1208622)

15 Hayashi T, Hirshman MF, Fujii N, Habinowski SA, Witters LA \& Goodyear LJ. Metabolic stress and altered glucose transport: activation of AMP-activated protein kinase as a unifying coupling mechanism. Diabetes 200049 527-531. (doi:10.2337/diabetes. 49.4.527)

16 Schlumberger M. Papillary and follicular thyroid carcinoma. New England Journal of Medicine 2000338 297-306. (doi:10.1056/ NEJM199801293380506)

17 Mazzaferri EL \& Massoll N. Management of papillary and follicular (differentiated) thyroid cancer: new paradigms using recombinant human thyrotropin. Endocrine-Related Cancer 20029 227-247. (doi:10.1677/erc.0.0090227)

18 Cooper DS, Doherty GM, Haugen BR, Kloos RT, Lee SL, Mandel SJ, Mazzaferri EL, McIver B, Pacini F, Schlumberger $\mathrm{M}$ et al. Revised American Thyroid Association management guidelines for patients with thyroid nodules and differentiated thyroid cancer. Thyroid 200919 1167-1214. (doi:10.1089/thy.2009.0110)

19 Goretzki PE, Simon D, Frilling A, Witte J, Reiners C, Grussendorf M, Horster FA \& Röher HD. Surgical reintervention for differentiated thyroid carcinoma. British Journal of Surgery 199480 1009-1012. (doi:10.1002/bjs.1800800826)

20 Haugen BR. Management of the patient with progressive radioiodine non-responsive disease. Seminars in Surgical Oncology 1999 16 34-41. (doi:10.1002/(SICI)1098-2388(199901/02)16:1<34:: AID-SSU7> 3.0.CO;2-2)

21 Coelho SM, Corbo R, Buescu A, Carvalho DP \& Vaisman M. Retinoic acid in patients with radioiodine non-responsive thyroid carcinoma. Journal of Endocrinological Investigation 2004 27 334-339.
22 De Lellis AR, Lloyd RV, Heitz PU \& Eng C. Thyroid and parathyroid tumors. In Pathology and Genetics of Tumors of Endocrine Organs. World Health Organization Classification of Tumors, 1st edn, pp 49-133. Eds AR De Lellis \& ED Williams. Lyon: WHO, IARC, 2004.

23 Leake R, Barnes D, Pinder S, Ellis I, Anderson L, Anderson T, Adamson R, Rhodes T, Miller K \& Walker R. Immunohistochemical detection of steroid receptors in breast cancer: a working protocol. UK Receptor Group, UK NEQAS, The Scottish Breast Cancer Pathology Group, and The Receptor and Biomarker Study Group of the EORTC. Journal of Clinical Pathology 200053 634-635. (doi:10.1136/jcp.53.8.634)

24 Wang W, Larson SM, Tuttle RM, Kalaigian H, Kolbert K, Sonenberg M \& Robbins RJ. Resistance of [18F]-fluorodeoxyglucose-avid metastatic thyroid cancer lesions to treatment with high-dose radioactive iodine. Thyroid 200112 1169-1175. (doi:10.1089/10507250152741028)

25 Faustino A, Couto JP, Pópulo H, Rocha AS, Pardal F, CameselleTeijeiro JM, Lopes JM, Sobrinho-Simões M \& Soares P. mTOR pathway overactivation in BRAF mutated papillary thyroid carcinoma. Journal of Clinical Endocrinology and Metabolism 2012 97 E1139-E1149. (doi:10.1210/jc.2011-2748)

26 Inoki K, Kim J \& Guan KL. AMPK and mTOR in cellular energy homeostasis and drug targets. Annual Review of Pharmacology and Toxicology 201210 381-400. (doi:10.1146/annurev-pharmtox010611-134537)

27 Esteve-Puig R, Canals F, Colomé N, Merlino G \& Recio JA. Uncoupling of the LKB1-AMPK $\alpha$ energy sensor pathway by growth factors and oncogenic BRAF. PLoS ONE 20094 e4771. (doi:10.1371/journal.pone.0004771)

28 de Souza EC, Padrón AS, Braga WM, de Andrade BM, Vaisman M, Nasciutti LE, Ferreira AC \& de Carvalho DP. MTOR downregulates iodide uptake in thyrocytes. Journal of Endocrinology $2010 \mathbf{2 0 6}$ 113-120. (doi:10.1677/JOE-09-0436)

29 Souza EC, Ferreira AC \& Carvalho DP. The mTOR protein as a target in thyroid cancer. Expert Opinion on Therapeutic Targets 2011 15 1099-10112. (doi:10.1517/14728222.2011.594044)

30 Schmid KW \& Farid NR. How to define follicular thyroid carcinoma? Virchows Archiv 2006448 385-393. (doi:10.1007/ s00428-006-0162-0)

31 Salt I, Celler JW, Hawley SA, Prescott A, Woods A, Carling D \& Hardie DG. AMP-activated protein kinase: greater AMP dependence, and preferential nuclear localization, of complexes containing the alpha2 isoform. Biochemical Journal 1998334 177-187.

Received 4 April 2013

Revised version received 27 June 2013

Accepted 29 July 2013 